\title{
Combining Contrast Information and Local Binary Patterns for Gender Classification
}

\author{
Juha Ylioinas, Abdenour Hadid, and Matti Pietikäinen \\ Machine Vision Group, P.O. Box 4500, \\ FI-90014 University of Oulu, Finland \\ \{juyl, hadid,mkp\}@ee.oulu.fi
}

\begin{abstract}
Recent developments in face analysis showed that local binary patterns (LBP) provide excellent results in representing faces. LBP is by definition a purely gray-scale invariant texture operator, codifying only the facial patterns while ignoring the magnitude of gray level differences (i.e. contrast). However, pattern information is independent of the gray scale, whereas contrast is not. On the other hand, contrast is not affected by rotation, but patterns are, by default. So, these two measures can supplement each other. This paper addresses how well facial images can be described by means of both contrast information and local binary patterns. We investigate a new facial representation which combines both measures and extensively evaluate the proposed representation on the gender classification problem, showing interesting results. Furthermore, we compare our results against those of using Haar-like features and AdaBoost learning, demonstrating improvements with a significant margin.
\end{abstract}

Keywords: Texture Features, Local Binary Patterns, Contrast, Gender Classification.

\section{Introduction}

Recent developments in face analysis showed that local binary patterns (LBP) 1 provide excellent results in representing faces [23]. For instance, it has been successfully applied to face detection 4, face recognition [2, facial expression recognition [5], gender classification [6] etc. LBP is a gray-scale invariant texture operator which labels the pixels of an image by thresholding the neighborhood of each pixel with the value of the center pixel and considers the result as a binary number. LBP labels can be regarded as local primitives such as curved edges, spots, flat areas etc. The histogram of the labels can be then used as a face descriptor. Due to its discriminative power and computational simplicity, the LBP methodology has already attained an established position in face analysis research 1 .

LBP is by definition a purely gray-scale invariant texture operator, codifying only the facial patterns while ignoring the magnitude of gray level differences (i.e.

\footnotetext{
${ }^{1}$ See LBP bibliography at http://www.cse.oulu.fi/MVG/LBP_Bibliography
} 
contrast). However, texture can be regarded as a two-dimensional phenomenon characterized by two orthogonal properties: spatial structure (patterns) and contrast (the strength of the patterns). Pattern information is independent of the gray scale, whereas contrast is not. On the other hand, contrast is not affected by rotation, but patterns are, by default. These two measures supplement each other in a very useful way. The question which arises then is how well facial images can be described by means of both contrast information and local binary patterns? In other words, could contrast information enhance the effectiveness of the popular LBP-based facial representation? If so, how the two measures could optimally be combined? This paper addresses these issues by proposing and investigating a new facial representation which combines contrast information and local binary patterns. We extensively evaluate the proposed facial representation on the gender classification problem, although other face related tasks such as face detection, facial expression recognition or face authentication could also be considered.

Gender classification from facial information consists of determining whether the person whose face is in the given image or video is a man or a woman. This is a two-class pattern recognition task which is very useful in many applications such as more affective Human-Computer Interaction (HCI), audience measurement and reporting, consumer behavior analysis and marketing, content-based image and video retrieval, restricting access to certain areas based on gender, and so on. The main challenges in gender classification from facial images are due to geometrical non-uniformity, make-up and occlusions, pose and illumination variations, and image degradations e.g. caused by blur and noise. These factors are unfortunately often present in real-world face images captured in unconstrained environments.

First attempts of using computer vision based techniques to gender classification started in early 1990s. Since then, a significant progress has been made and several approaches have been reported in literature. Fundamentally, the proposed techniques differ in (i) the choice of the facial representation, ranging from the use of simple raw pixels to more complex features such as Gabor responses, and in (ii) the design of the classifier, ranging from the use of nearest neighbor (NN) and fisher linear discriminant (FLD) classifiers to artificial neural networks (ANN), support vector machines (SVM) and boosting schemes. For instance, Moghaddam and Yang [7] used raw pixels as inputs to SVMs while Baluja and Rowley [8] adopted AdaBoost to combine weak classifiers, constructed using simple pixel comparisons, into single strong classifier. Both systems showed good classification rates. A comparative analysis on gender classification approaches can be found in 9 .

The rest of this paper is organized as follows. Section 2 briefly describes the popular LBP methodology for representing face images. Our proposed facial representation that combines contrast information and local binary patterns is then presented in Section 3. Section 4 considers the gender classification problem and provide extensive experiments and analysis on the effectiveness of using contrast information to supplement local binary patterns. The section also analyzes the 
effects of illumination normalization, the generalization ability and the real-time implementation of the proposed gender classification method. A conclusion is drawn in Section 5 .

\section{Face Representation Using Local Binary Patterns}

The LBP texture analysis operator, introduced by Ojala et al. [1], is defined as a gray-scale invariant texture measure, derived from a general definition of texture in a local neighborhood. It is a powerful means of texture description and among its properties in real-world applications are its discriminative power, computational simplicity and tolerance against monotonic gray-scale changes.

The original LBP operator forms labels for the image pixels by thresholding the $3 \times 3$ neighborhood of each pixel with the center value and considering the result as a binary number. Fig. 1 shows an example of an LBP calculation. The histogram of these $2^{8}=256$ different labels can then be used as a texture descriptor.

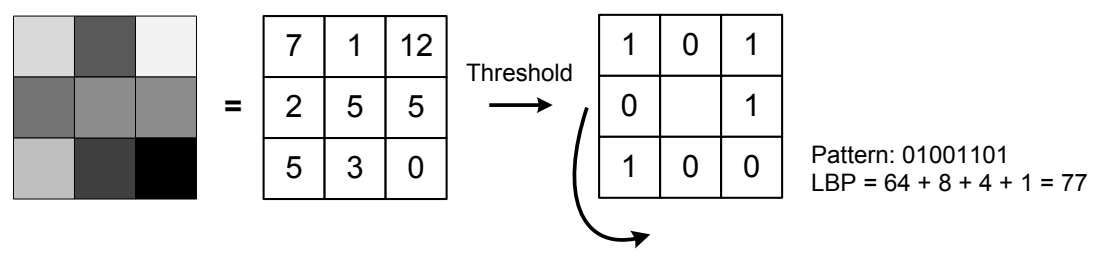

Fig. 1. The basic LBP operator

The operator has been extended to use neighborhoods of different sizes. Using a circular neighborhood and bilinearly interpolating values at non-integer pixel coordinates allow any radius and number of pixels in the neighborhood. The notation $(P, R)$ is generally used for pixel neighborhoods to refer to $P$ sampling points on a circle of radius $R$. The calculation of the LBP codes can be easily done in a single scan through the image. The value of the LBP code of a pixel $\left(x_{c}, y_{c}\right)$ is given by:

$$
\operatorname{LBP}_{P, R}=\sum_{p=0}^{P-1} s\left(g_{p}-g_{c}\right) 2^{p},
$$

where $g_{c}$ corresponds to the gray value of the center pixel $\left(x_{c}, y_{c}\right), g_{p}$ refers to gray values of $P$ equally spaced pixels on a circle of radius $R$, and $s$ defines a thresholding function as follows:

$$
s(x)=\left\{\begin{array}{l}
1, \text { if } x \geq 0 ; \\
0, \text { otherwise. }
\end{array}\right.
$$

Another extension to the original operator is the definition of so called uniform patterns. This extension was inspired by the fact that some binary patterns occur 
more commonly in texture images than others. A local binary pattern is called uniform if the binary pattern contains at most two bitwise transitions from 0 to 1 or vice versa when the bit pattern is traversed circularly. In the computation of the LBP labels, uniform patterns are used so that there is a separate label for each uniform pattern and all the non-uniform patterns are labeled with a single label. This yields to the following notation for the $\operatorname{LBP}$ operator: $\operatorname{LBP}_{P, R}^{u 2}$. The subscript represents using the operator in a $(P, R)$ neighborhood. Superscript $u 2$ stands for using only uniform patterns and labeling all remaining patterns with a single label.

Each LBP label (or code) can be regarded as a micro-texton. Local primitives which are codified by these labels include different types of curved edges, spots, flat areas etc. The occurrences of the LBP codes in the image are collected into a histogram. The classification is then performed by computing histogram similarities. For an efficient representation, facial images are first divided into several local regions from which LBP histograms are extracted and concatenated into an enhanced feature histogram.

\section{Face Representation Using LBP and Contrast}

LBP operator by itself totally ignores the contrast information which is a property of texture usually regarded as a very important cue for our vision system. In many applications, a purely gray-scale invariant texture operator like LBP may waste useful information, and adding gray-scale dependent information like contrast may enhance the accuracy of the method. This observation is behind our idea of combining contrast information and local binary patterns for facial representation.

We measure the rotation invariant local contrast in a circularly symmetric neighbor set just like the LBP:

$$
\operatorname{VAR}_{P, R}=\frac{1}{P} \sum_{p=0}^{P-1}\left(g_{p}-\mu\right)^{2}, \quad \text { where } \quad \mu=\frac{1}{P} \sum_{p=0}^{P-1} g_{p} .
$$

$\mathrm{VAR}_{P, R}$ is, by definition, invariant against shifts in the gray scale. Since contrast is measured locally, the measure can resist even intra-image illumination variation as long as the absolute gray value differences are not badly affected.

Like in LBP methodology, the contrast measures could be collected into a histogram and used as a contrast descriptor of the face. However, variance measure has a continuous-valued output; hence, quantization of its feature space is needed before computing the histograms. We therefore perform the quantization by adding together feature distributions for every single model image in a total distribution, which is then divided into $B$ bins having an equal number of entries, thus obtaining the cut values of the bins of the histograms. In our experiments, we have set the value of $B$ to 8 . Fig. 2 shows some examples of contrast images after quantization.

After quantization, we collect the contrast measures into a histogram as follows: we first divide the face into blocks and then extract a contrast histogram 


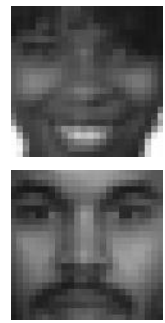

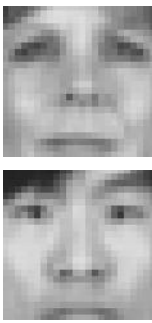

(a)
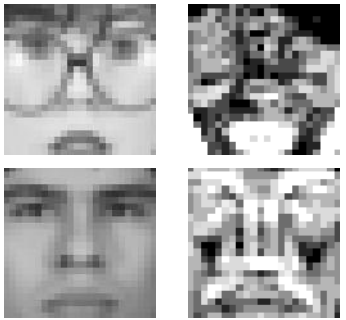

(b)

Fig. 2. Examples of contrast images using VAR operator. (a) Original training samples and (b) corresponding contrast images using $\mathrm{VAR}_{8,1}$ operator and $B=8$ level of quantization.

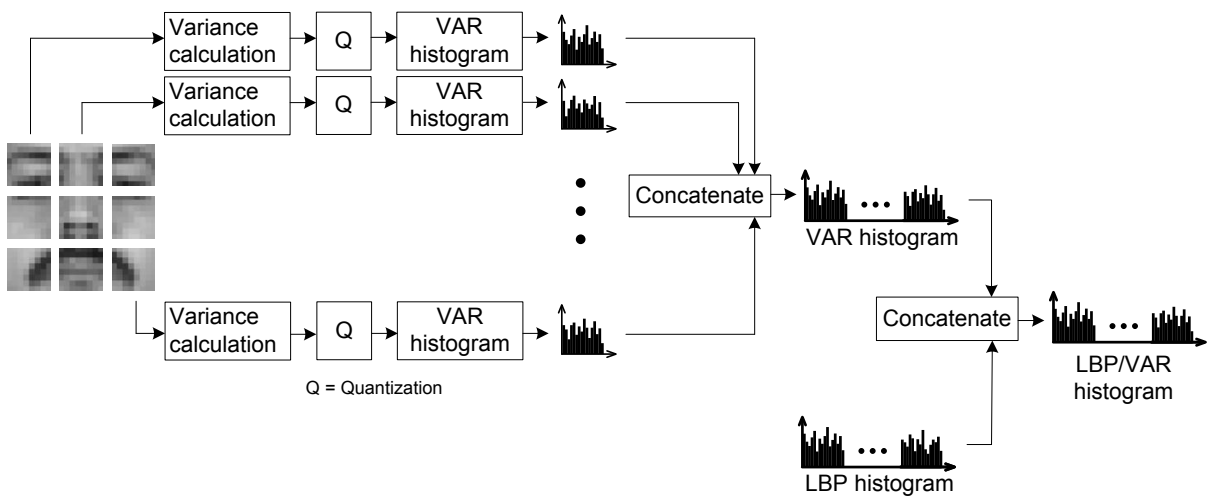

Fig. 3. Proposed facial representation combining contrast information and local binary patterns

from each block and concatenate them into a single histogram that is used as a contrast descriptor of the face.

Hence, given a facial image, our proposed representation for describing both facial texture patterns (LBP) and their strength (i.e. contrast) consists of (i) dividing the face into local blocks; (ii) extracting local LBP histograms and concatenating them into a single LBP histogram; (iii) extracting local contrast histograms and concatenating them into a single contrast histogram; and (iv) concatenating the LBP and contrast histograms to obtain spatially enhanced histogram denoted as LBP/VAR histogram. The procedure of extracting the $\mathrm{LBP} / \mathrm{VAR}$ histogram is illustrated in Fig. 3 .

\section{Experiments on Gender Classification}

To assess the effectiveness of combining contrast information and local binary patterns, we considered the gender classification problem and conducted 
extensive experiments evaluating the performance of the proposed facial representation and comparing the results against those of using only LBP (i.e. without contrast).

\subsection{Experimental Data}

For experiments we considered three publicly available face databases namely FRGC 2.0 [10, FERET [1], and XM2VTS [12]. The databases encompass variations in illumination, expressions, pose angles, age of the subjecs, ethnicity etc. We randomly divided the datasets into training and testing sets as described in Table 1 .

Table 1. The division of the data into training and testing sets

\begin{tabular}{|l|c|c|c||c|c|c|}
\hline \multirow{2}{*}{ Database } & \multicolumn{3}{|c||}{ Training } & \multicolumn{3}{c|}{ Testing } \\
\cline { 2 - 7 } & Males & Females & Total & Males & Females & Total \\
\hline FRGC 2.0 & 13,565 & 10,518 & 24,083 & 2,050 & 2,050 & 4,100 \\
\hline FERET & 1,801 & 1,039 & 2,840 & 260 & 260 & 520 \\
\hline XM2VTS & 1,092 & 962 & 2,054 & 130 & 130 & 230 \\
\hline
\end{tabular}

\subsection{Settings}

We first normalized the training face samples to $24 \times 24$ pixels using the eye coordinates that are supplied with the datasets. Figure 4 illustrates some examples from the training samples.

Our proposed facial representation involves the following free parameters to be fixed: the number and size of blocks when dividing the face images, the radius and number of neighbors for the LBP operator, the radius and number of neighbors for the contrast operator (VAR) and the quantization level.

Regarding the division of the faces into blocks and given the relatively small size of the training face images $(24 \times 24$ pixels), we divided the images into $3 \times 3$ equally sized rectangular regions as shown in Figure 3 . We considered both overlapping (using 3 pixels both horizontally and vertically) and non-overlapping
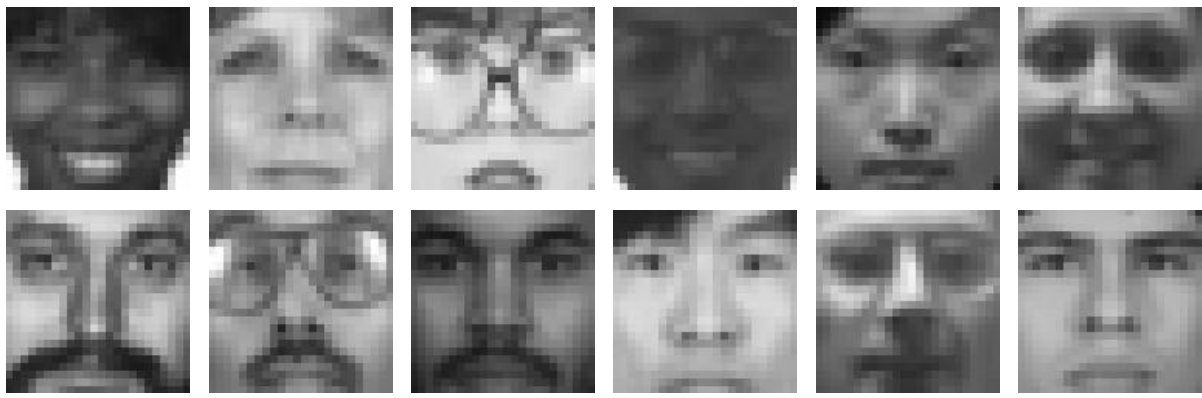

Fig. 4. Sample images from the training set 
divisions, yielding block sizes of $10 \times 10$ pixels for overlapped blocks and $8 \times 8$ pixels for non-overlapping blocks.

When looking for the optimal LBP operator, we noticed that LBP representation is quite robust with respect to the selection of $P$ and $R$ parameters. Changes in these parameters may cause big differences in the length of the feature vector, but the overall performance is not necessarily affected significantly. Hence, we selected the $\operatorname{LBP}_{8,1}^{u 2}$ operator since it is a good trade-off between discriminative power and feature vector length.

For contrast calculation, we used the rotation invariant variance $\mathrm{VAR}_{P, R}$ operator with 8 neighbors $(P=8)$ in radius of $1(R=1)$. Given the continuous-valued nature of the contrast measures, quantization is then needed before computing the histograms. We first computed a total contrast distribution from all our training samples by adding together feature distributions from each sample. We then performed quantization by thresholding the total distribution into 8 sections having an equal number of entries. Hence, we determined the threshold values that are later used for quantizing the contrast measures for the test samples. Therefore, in most experiments $\mathrm{LBP}_{8,1}^{u 2}$ and $\mathrm{VAR}_{8,1}$ operators are used for computing the histograms.

For determining the gender of a person in a given test image, we used the extracted facial representations as inputs to an SVM classifier with a non-linear RBF kernel. The choice of SVM is motivated by its proven performance in various object detection and recognition tasks in computer vision. The parameters of the SVM classifier were determined using grid search and five-fold cross validation. For SVM implementation, we used the publically available LIBSVM library [13].

For the purpose of analyzing the effects of illumination variations, we also considered an illumination normalization procedure using Tan and Triggs' method [14.

\subsection{Results and Analysis}

We conducted extensive experiments in 9 different configurations. Tables 2 and 3 describe the considered parameters in each configuration. In the first seven experiments (\#1 till \#7), we trained the classifier using only training samples from FRGC 2.0 database and evaluated the performance on the rest of the data including FRGC 2.0, FERET and XM2VTS test samples. In experiments \#8 and $\# 9$, the system was trained using training samples from FRGC 2.0 and FERET databases, and evaluated on FRGC 2.0, FERET and XM2VTS test data. Training the system on one or more databases and then doing evaluation on other (different) databases is important for gaining insight into the generalization ability of the proposed method under different and unknown conditions.

The average classification rates in each configuration are summarized in the last columns of Tables 2 and 3 , whereas more detailed results are given in Table 4.

(A) Importance of contrast information: The primary goal of the experiments was to analyze whether facial images can be efficiently described by means of both contrast information and local binary patterns. In other terms, could contrast information enhance the effectiveness of the popular LBP-based 
Table 2. Experiments conducted using FRGC 2.0 training samples

\begin{tabular}{|c|l|c|c|c|c|c|}
\hline $\begin{array}{c}\text { Exp. } \\
(\#)\end{array}$ & Method & $\begin{array}{c}\text { Window } \\
\text { size }\end{array}$ & Overlap & $\begin{array}{c}\text { Illumination } \\
\text { normalization }\end{array}$ & $\begin{array}{c}\text { Quantization } \\
\text { levels }\end{array}$ & $\begin{array}{c}\text { Avg. classification } \\
\text { rate }(\%)\end{array}$ \\
\hline 1 & $\mathrm{LBP}_{4,1}$ & $10 \times 10$ & 3 & - & - & 87.34 \\
\hline 2 & $\mathrm{LBP}_{8,1}^{u 2}$ & $8 \times 8$ & - & - & - & 91.53 \\
\hline 3 & $\mathrm{LBP}_{8,1}^{u 2}$ & $10 \times 10$ & 3 & - & - & 91.93 \\
\hline 4 & $\mathrm{LBP}_{8,1}^{u 2}$ & $8 \times 8$ & - & applied & - & 92.13 \\
\hline 5 & $\mathrm{LBP}_{8,1}^{u 2}$ & $10 \times 10$ & 3 & applied & - & 91.69 \\
\hline 6 & $\mathrm{LBP}_{8,1}^{u 2} / \mathrm{VAR}_{8,1}$ & $8 \times 8$ & - & - & 8 & $\mathbf{9 4 . 5 3}$ \\
\hline 7 & $\mathrm{LBP}_{8,1}^{u 2} / \mathrm{VAR}_{8,1}$ & $10 \times 10$ & 3 & - & 8 & 93.73 \\
\hline
\end{tabular}

Table 3. Experiments conducted using FRGC 2.0 and FERET training samples

\begin{tabular}{|c|l|c|c|c|c|c|}
\hline $\begin{array}{c}\text { Exp. } \\
(\#)\end{array}$ & Method & $\begin{array}{c}\text { Window } \\
\text { size }\end{array}$ & Overlap & $\begin{array}{c}\text { Illumination } \\
\text { normalization }\end{array}$ & $\begin{array}{c}\text { Quantization } \\
\text { levels }\end{array}$ & $\begin{array}{c}\text { Avg. classification } \\
\text { rate }(\%)\end{array}$ \\
\hline 8 & $\mathrm{LBP}_{8,1}^{u 2}$ & $10 \times 10$ & 3 & - & - & 92.31 \\
\hline 9 & $\mathrm{LBP}_{8,1}^{u 2} / \mathrm{VAR}_{8,1}$ & $10 \times 10$ & 3 & - & 8 & $\mathbf{9 6 . 3 3}$ \\
\hline
\end{tabular}

facial representation? The obtained results comparing LBP versus LBP/VAR (see Exps. \#2 versus \#6, \#3 versus \#7, and \#8 versus \#9) clearly indicate that combining contrast information and local binary patterns does enhance the gender classification performance in all configurations. The performance gain was around $3 \%$.

(B) Effects of illumination normalization: To gain insight into the sensitivity of the proposed facial representations against illumination variations, we considered and experimented with an illumination normalization procedure proposed by Tan and Triggs [14. It consists of pre-processing the facial images by applying Gamma correction, difference of Gaussian (DoG) filtering, masking and equalization. The experiments (see Exps. \#2 versus \#4 and \#3 versus \#5) showed no significant improvements using illumination normalization, hence pointing out the relative robustness of our proposed approach against illumination variations.

(C) Generalization ability of the system: To gain insight into the generalization ability of the system under different and unknown conditions, we considered experiments in which training and test samples are taken from different databases. As expected, the results showed performance degradation when evaluating the system on different and unknown conditions. This problem can be alleviated by training the system on larger and different databases. For instance, training the system using samples from only FRGC database yielded classification rate of $93.73 \%$ while using training samples from both FRGC and FERET databases improved the performance, reaching $96.33 \%$.

(D) Comparison to other methods: We compared our results against those of one of the state-of-the-art methods using Haar-like features and AdaBoost learning for gender classification. The obtained results, shown in Table 5 clearly 
Table 4. Comparison of gender classification results of several approaches on three different test sets

\begin{tabular}{|c|c|c|c|c|c|c|c|}
\hline \multirow{2}{*}{$\begin{array}{c}\text { Exp. } \\
(\#)\end{array}$} & \multirow{2}{*}{ Method } & \multicolumn{5}{|c|}{ Gender Classification Rate (\%) } \\
\cline { 3 - 8 } & & \multicolumn{2}{|c|}{ FRGC 2.0 } & \multicolumn{2}{|c|}{ FERET } & \multicolumn{2}{|c|}{ XM2VTS } \\
\cline { 3 - 8 } & Males & Females & Males & Females & Males & Females \\
\hline 1 & $\mathrm{LBP}_{4,1}$ & 91.95 & 89.22 & 73.46 & 71.46 & 82.31 & 56.15 \\
\hline 2 & $\mathrm{LBP}_{8,1}^{u 2}$ & 94.63 & 93.17 & 88.46 & 73.08 & 90.77 & 60.77 \\
\hline 3 & $\mathrm{LBP}_{8,1}^{u 2}$ & 95.51 & 93.22 & 85.38 & 74.62 & 86.15 & 68.46 \\
\hline 4 & $\mathrm{LBP}_{8,1}^{u 2}$ & 95.80 & 93.37 & 90.77 & 70.77 & 83.85 & 68.43 \\
\hline 5 & $\mathrm{LBP}_{8,1}^{u 2}$ & 95.29 & 93.71 & 88.08 & 66.15 & 83.58 & 70.00 \\
\hline 6 & $\mathrm{LBP}_{8,1}^{u 2} / \mathrm{VAR}_{8,1}$ & 98.00 & 96.97 & 87.69 & 73.46 & 92.31 & 59.23 \\
\hline 7 & $\mathrm{LBP}_{8,1}^{u 2} / \mathrm{VAR}_{8,1}$ & 98.78 & 94.39 & 89.62 & 68.46 & 93.08 & 63.08 \\
\hline \hline 8 & $\mathrm{LBP}_{8,1}^{u 2}$ & 92.98 & 94.44 & 88.38 & 84.62 & 89.23 & 74.62 \\
\hline \hline 9 & $\mathrm{LBP}_{8,1}^{u 2} / \mathrm{VAR}_{8,1}$ & 98.24 & 96.98 & 95.38 & 87.69 & 90.77 & 80.77 \\
\hline
\end{tabular}

Table 5. Comparison between our proposed method and Haar-classifier based on AdaBoost learning

\begin{tabular}{|c|c|c|c|c|c|c|c|c|c|c|}
\hline \multirow{3}{*}{ Method } & \multicolumn{10}{|c|}{ Gender Classification Rate (\%) } \\
\hline & \multicolumn{3}{|c|}{ FRGC 2.0 } & \multicolumn{3}{|c|}{ FERET } & \multicolumn{3}{|c|}{ XM2VTS } & All \\
\hline & $\mathrm{M}$ & $\mathrm{F}$ & Avg. & $\mathrm{M}$ & $\mathrm{F}$ & Avg. & $\mathrm{M}$ & $\mathrm{F}$ & Avg. & Avg. \\
\hline $\mathrm{LBP}_{8,1}^{u 2} / \mathrm{VAR}_{8,1}+\mathrm{SVM}_{(\mathrm{Exp} . ~ \# 9)}$ & 98.24 & 96.98 & 97.61 & 95.38 & 87.69 & 91.54 & 90.77 & 80.77 & 85.77 & 96.33 \\
\hline Haar-like features + AdaBoost & 79.71 & 82.93 & 81.32 & 86.92 & 76.15 & 81.54 & 76.15 & 77.69 & 76.92 & 81.10 \\
\hline
\end{tabular}

assess the effectiveness of our proposed approach as it outperforms the method using Haar-like features and AdaBoost with a significant margin (96.33\% versus $81.10 \%)$.

(E) Real-time implementation: Among the advantages of using LBP-like facial representation is the computational simplicity of the LBP operator. We built a real-time demonstration using the LBP representation and SVM for gender classification in real-world scenarios. Including face and eye detection modules, the framework runs at more than 17 frames per second on a $3 \mathrm{GHz}$ Intel Core 2 Duo computer and successfully recognizes the gender of the users in most cases.

\section{Conclusion}

From the observation that LBP approach codifies only the facial patterns while ignoring their strength, we proposed a novel facial representation combining LBP and contrast information. The extensive experiments on the gender classification problem showed significant performance enhancement compared to popular methods such as basic LBP method or using Haar-like features with AdaBoost learning. Pre-processing the facial images using illumination normalizations seemed to not enhance the performance, hence pointing out the relative robustness of our proposed approach against illumination variations. To 
gain insight into the generalization ability of the proposed approach, we considered experiments in which training and test samples are taken from different databases. The results suggested using larger and different databases to alleviate the generalization problem. Exploiting the computational simplicity of the LBPlike facial representations, we also built a real time demonstration for real-world applications.

Analyzing the misclassification errors made by the system, we noticed that female's faces are harder to classify than male's ones. Perhaps, this could be explained by the fact that when only facial areas are used for gender classification, the presence of moustaches and beards helps more the classification of male's images. However, one can expect better classification of female's images when external features such as hair are also included. This issue will be further investigated in our future work.

To further assess the effectiveness of combining contrast information and local binary patterns for face representation, we also plan to extend the evaluation of our methodology on other face-related tasks including age estimation and ethnicity classification especially from real-life faces acquired in unconstrained conditions using, for instance, the recently built database called the Labeled Faces in the Wild (LFW) 15.

\section{Acknowledgment}

This work has been partially performed within two EU funded projects called MOBIO (contract number: IST-214324) and TABULA RASA (grant agreement number: 257289). These projects are within the 7th Framework Research Programme of the European Union (EU).

\section{References}

1. Ojala, T., Pietikäinen, M., Mäenpää, T.: Multiresolution gray-scale and rotation invariant texture classification with local binary patterns. IEEE Transactions on Pattern Analysis and Machine Intelligence 24(7), 971-987 (2002)

2. Ahonen, T., Hadid, A., Pietikäinen, M.: Face description with local binary patterns: Application to face recognition. IEEE Transactions on Pattern Analysis and Machine Intelligence 28(12), 2037-2041 (2006)

3. Hadid, A., Zhao, G., Ahonen, T., Pietikäinen, M.: Face analysis using local binary patterns. In: Mirmehdi, M., Xie, X., Suri, J. (eds.) Handbook of Texture Analysis, pp. 347-373. Imperial College Press, London

4. Hadid, A., Pietikäinen, M., Ahonen, T.: A discriminative feature space for detecting and recognizing faces. In: IEEE Conference on Computer Vision and Pattern Recognition, vol. II, pp. 797-804 (2004)

5. Shan, C., Gong, S., McOwan, P.: Facial expression recognition based on local binary patterns:a comprehensive study. Image and Vision Computing 27(6), 803-816 (2009)

6. Yang, Z., Ai, H.: Demographic classification with local binary patterns. In: International Conference on Biometrics, pp. 464-473 (2007) 
7. Moghaddam, B., Yang, M.H.: Learning gender with support faces. IEEE Transactions on Pattern Analysis and Machine Intelligence 24(5), 707-711 (2002)

8. Baluja, S., Rowley, H.: Boosting sex identification performance. International Journal of Computer Vision 71, 111-119 (2007)

9. Mäkinen, E., Raisamo, R.: Evaluation of gender classification methods with automatically detected and aligned faces. IEEE Transactions on Pattern Analysis and Machine Intelligence 30(3), 541-547 (2008)

10. Phillips, P.J., Flynn, P.J., Scruggs, T., Bowyer, K.W., Chang, J., Hoffman, K., Marques, J., Min, J., Worek, W.: Overview of the face recognition grand challenge. In: IEEE Computer Society Conference on Computer Vision and Pattern Recognition 2005 (CVPR 2005), pp. 947-954 (2005)

11. Phillips, P.J., Wechsler, H., Huang, J., Rauss, P.: The FERET database and evaluation procedure for face recognition algorithms. Image and Vision Computing 16(10), 295-306 (1998)

12. Messer, K., Matas, J., Kittler, J., Luettin, J., Maitre, G.: XM2VTSDB: The extended M2VTS database. In: Second International Conference on Audio- and Video-based Biometric Person Authentication 1999 (AVBPA 1999), pp. 72-77 (1999)

13. Chang, C.-C., Lin, C.-J.: LIBSVM: a library for support vector machines (2001), Software, available at http://www.csie.ntu.edu.tw/ cjlin/libsvm

14. Tan, X., Triggs, B.: Enhanced local texture feature sets for face recognition under difficult lighting conditions. IEEE Transactions on Image Processing 19(6), 16351650 (2010)

15. Huang, G.B., Ramesh, M., Berg, T., Learned-Miller, E.: Labeled Faces in the Wild: A Database for Studying Face Recognition in Unconstrained Environments. University of Massachusetts, Amherst, Technical Report 07-49 (2007) 